(2) Open Access Full Text Article

\title{
Assessing the association between omalizumab and arteriothrombotic events through spontaneous adverse event reporting
}

This article was published in the following Dove Press journal:

Journal of Asthma and Allergy

27 April 2012

Number of times this article has been viewed

\author{
Ayad K Ali \\ Abraham G Hartzema \\ Department of Pharmaceutical \\ Outcomes and Policy, College of \\ Pharmacy, University of Florida, \\ Gainesville, FL, USA
}

Correspondence: Ayad K Ali

I0I S Newell Drive, PO Box 100496 ,

Gainesville, FL 326I0, USA

Tel +l 3522736629

$\mathrm{Fax}+\mathrm{I} 3522736270$

Email ayadali@ufl.edu
Background: Omalizumab is a monoclonal antibody, indicated for the treatment of severe allergic asthma. In Europe, there have been concerns about the cardiovascular safety of omalizumab. The objective of this study was to analyze the association between omalizumab and arterial thrombotic events in a spontaneous adverse drug reaction reporting database in the US.

Methods and materials: Reports of arterial thrombotic events submitted to the US Food and Drug Administration's Adverse Event Reporting System (AERS) between 2004 and 2011 were retrieved and analyzed by the reporting odds ratio data mining algorithm. The reporting odds ratio of arterial thrombotic events for omalizumab was compared with specific asthma medications and all drugs in the AERS. Values $\geq 2$ were considered significant safety signals. The Medical Dictionary for Regulatory Activities Preferred Terms were used to identify arterial thrombotic events (eg, stroke, myocardial infarction).

Results: In total, 293,783 reports of arterial thrombotic events were retrieved (about $2 \%$ of all adverse drug reaction reports), corresponding to 2274 asthma drug-arterial thrombotic events pairs (omalizumab, 222; inhaled corticosteroids [ICS], 131; long-acting beta-agonists [LABA], 102; single-device combination ICS-LABA, 506; inhaled short-acting beta-agonists [SABA], 475; oral SABA, 6; inhaled antimuscarinics [AMC], 477; single-device combination AMC-SABA, 127; xanthines, 50; leukotriene modifiers, 174; and mast cell stabilizers, 4). Reporting odds ratio and 95\% confidence interval values for omalizumab compared with other asthma drugs and all drugs in AERS were 2.75 (2.39-316) and 1.09 (0.95-1.24), respectively. Omalizumab ranked second after ICS in the risk of arterial thrombotic events, followed by AMC, AMC-SABA, and ICS-LABA.

Conclusion: Omalizumab is associated with higher than expected reporting of arterial thrombotic events in asthmatic patients. This hypothesis needs further testing in robust epidemiological studies.

Keywords: omalizumab, postmarketing safety surveillance, adverse event reporting system, arterial thrombotic events

\section{Introduction}

Omalizumab is a monoclonal antibody that blocks immunoglobulin E, thereby inhibiting the early and late inflammatory reactions of asthma. ${ }^{1}$ On June 20, 2003, omalizumab (Xolair ${ }^{\mathbb{B}}$; Genentech Inc, San Francisco, CA) was approved by the US Food and Drug Administration (FDA) for the treatment of severe persistent allergic asthma refractory to standard treatment. ${ }^{2}$ The drug is available in $75 \mathrm{mg}$ and $150 \mathrm{mg}$ powder and a solvent for injection, which is administered subcutaneously to patients aged 6 years or older, and the dose can be repeated every $2-4$ weeks. $^{3}$ 
In February 2011, the UK Medicines and Healthcare Products Regulatory Agency advised prescribers to be vigilant about the possible occurrence of arterial thrombotic events in patients exposed to omalizumab, and to report suspected relevant adverse reactions to the UK spontaneous adverse event reporting system (ie, the Yellow Card Scheme).,5 These recommendations were based on interim analyses of randomized clinical trials and an unpublished cohort study, ie, EXCELS (Evaluating Clinical Effectiveness and LongTerm Safety in patients with Moderate-to-Severe Asthma)., ${ }^{4,6}$ These analyses yielded a statistically insignificant increase in the risk of arterial thrombotic events in groups exposed to omalizumab compared with their unexposed control counterparts (randomized clinical trials: hazard ratio [HR] 1.86, 95\% confidence interval [CI] 0.73-4.72); EXCELS: HR 1.11, 95\% CI $0.70-1.76){ }^{4,6}$ The FDA reviewed the reported adverse events for omalizumab in July 2009, and concluded with a warning to prescribers and patients about hypersensitivity reactions, with no reference to any arteriothrombotic risks. ${ }^{?}$ This study assessed the safety profile of omalizumab with respect to arterial thrombotic events recorded in the FDA Adverse Event Reporting System (AERS) database.

\section{Methods and materials}

\section{Data source}

Adverse event reports recorded in FDA AERS from January 1, 2004 to January 1, 2011 were downloaded from the FDA website (www.fda.gov). ${ }^{8}$ The AERS contains reports of adverse drug events spontaneously submitted by physicians, pharmacists, other health care professionals, manufacturers, and consumers from the US and other countries. The Medical Dictionary for Regulatory Activities (MedDRA) Preferred Terms were used to codify the adverse events. Reports for the same patient but from different reporting sources (duplicate reports) were identified and excluded from the analysis. The structure of the AERS database is described elsewhere. ${ }^{9}$

\section{Identifying omalizumab and other asthma drugs}

Omalizumab and other asthma drugs were identified by their nonproprietary and brand names, which in most cases were recorded verbatim from individual adverse event reports (Table 1). In addition to omalizumab, identified classes of asthma drugs included: inhaled corticosteroids (ICS); longacting beta-agonists (LABA); single-device combination ICSLABA; inhaled short-acting beta-agonists (SABA); orally administered SABA; inhaled antimuscarinics (AMC); singledevice combination SABA-AMC; oral xanthines; leukotriene
Table I Identified asthma drugs

\begin{tabular}{lll}
\hline Drug class & Individual agent & Product example* \\
\hline $\begin{array}{l}\text { Monoclonal } \\
\text { immunoglobulin }\end{array}$ & Omalizumab & Xolair \\
E antibody & & \\
ICS & Beclomethasone & Beclovent \\
& Budesonide & Pulmicort \\
& Fluticasone & Flovent \\
Inhaled LABA & Mometasone & Asmanex \\
& Formoterol & Foradil \\
Single-device & Salmeterol & Serevent \\
ICS-LABA & Fudesonide-formoterol & Symbicort \\
& Mometasone-formoterol & Advair \\
Inhaled SABA & Salbutamol (albuterol) & Ventolin \\
Oral SABA & Salbutamol (albuterol) & Proventil \\
Inhaled AMC & Ipratropium & Atrovent \\
& Oxitropium & Oxivent \\
Single-device & Tiotropium & Spiriva \\
SABA-AMC & Salbutamol-ipratropium & Combivent \\
Oral xanthines & Theophylline & \\
& Aminophylline & Theovent \\
Leukotriene & Montelukast & Phyllocontin \\
modifiers & Zafirlukast & Singulair \\
& Zileuton & Accolate \\
Mast cell & Nedocromil & Zyflo \\
stabilizers & Cromolyn sodium & Tilade \\
\hline Note: & Intal \\
\hline
\end{tabular}

Note: *Product brand names are the property of their respective manufacturers. Abbreviations: AMC, antimuscarinics; ICS, inhaled corticosteroids; LABA, long-acting beta-agonists; SABA, short-acting beta-agonists.

modifiers; and mast cell stabilizers. In order to maximize the accuracy of capturing all the exposures of interest, the drug variable column of the AERS dataset was manually mined to extract the exposures of interest. The Clinical Pharmacology web-based reference tool (www.clinicalpharmacology.com) ${ }^{10}$ is used to verify the names of the reported products in the drug variable whenever the recorded names are confusing or unclear, which was the case for a few reports (3.2\%). Similarly, the drug name was crossreferenced with the corresponding indication variable column for the relevant report to look for asthma, chronic obstructive pulmonary disease, or respiratoryrelated clinical indications, whenever reported. Reports for drugs with other indications, unspecified or missing drug names, intravenously administered xanthines, and intranasally and ophthalmic administered corticosteroids and mast cell stabilizers, are excluded from the analysis.

\section{Identifying arterial thrombotic events}

Clinical judgment was applied to identify arterial thrombotic events, which were defined by the MedDRA Preferred Terms codes classified under the terms listed in Table 2. 
Table 2 Selected MedDRA Preferred Terms that reflect arterial thrombotic events

\section{MedDRA Preferred Terms category*}

Acute coronary syndrome

Angina

Ischemic heart disease

Myocardial infarction

Note: *Each term category has multiple verbatim terms identified.

Abbreviation: MedDRA, Medical Dictionary for Regulatory Activities.

A complete list of the identified codes is available upon request from the corresponding author. Reports with missing or unspecified adverse events were excluded from the study.

\section{Reporting odds ratio data mining algorithm}

The association between asthma drugs, including omalizumab, and arterial thrombotic events was analyzed using the reporting odds ratio and corresponding 95\% CI data mining algorithm. Reporting odds ratio values for omalizumab denote the ratio of the observed to expected number of reported arterial thrombotic events compared with other asthma drugs in the data set during the analysis period. Values $<1$ indicate no exposure-event associations; estimates $>1$ depict exposure-event safety signals. From the regulatory science perspective, safety signals are considered significant when the reporting odds ratio estimates and the lower limits of the corresponding $95 \% \mathrm{CI}$ are $\geq 2 .{ }^{11}$

\section{Data analysis}

Table 3 depicts the data mining algorithm to calculate the point estimates and the corresponding 95\% CI for omalizumab, which was applied for all other asthma drugs in the study. Data management and analyses were performed using SAS software for Windows (version 9.3; SAS Institute Inc, Cary, NC). The tables and figures were generated by Microsoft Office Word software (Microsoft Corporation,

Table 3 Reporting odds ratio and 95\% confidence interval data mining algorithm for omalizumab

\begin{tabular}{llll}
\hline Exposure & ATE & Other ADR & Total \\
\hline Omalizumab & $\mathrm{A}$ & $\mathrm{B}$ & $\mathrm{A}+\mathrm{B}$ \\
Other asthma drugs & $\mathrm{C}$ & $\mathrm{D}$ & $\mathrm{C}+\mathrm{D}$ \\
Total & $\mathrm{A}+\mathrm{C}$ & $\mathrm{B}+\mathrm{D}$ & $\mathrm{N}$ \\
$\mathrm{ROR}=\frac{\mathrm{A} \times \mathrm{D}}{\mathrm{C} \times \mathrm{B}} 95 \% \mathrm{Cl}=\mathrm{e}^{\left(\ln (\mathrm{ROR}) \pm 1.96 \sqrt{\frac{1}{\mathrm{~A}}+\frac{1}{\mathrm{~B}}+\frac{1}{\mathrm{C}}+\frac{1}{\mathrm{D}}}\right)}$ & \\
\hline
\end{tabular}

Abbreviations: ATE, arteriothrombotic events; $A D R$, adverse drug reactions; $C l$, confidence interval; ROR, reporting odds ratio.
Redmond, WA). Statistical significance tests were conducted at the alpha $=5 \%$ level.

\section{Results Overview of reports}

From January 2004 to January 2011, 15,519,543 adverse event reports were recorded in the AERS; about $2 \%$ involved asthma drugs $(280,857)$, and an additional $2 \%$ involved arterial thrombotic events $(293,782)$, corresponding to 2274 asthma drug-arterial thrombotic event pairs, with 222 (10\%) reports concerning omalizumab. Omalizumab ranked fourth in the number of arterial thrombotic event reports for asthma drugs, preceded by ICS-LABA, inhaled SABA, and AMC (Figure 1). Table 4 shows the distribution of identified specific arterial thrombotic events for asthma drugs. Myocardial infarction and stroke accounted for $29 \%$ and $27 \%$ of the arterial thrombotic event reports for omalizumab, respectively. Except for orally administered SABA and leukotriene modifiers, myocardial infarction contributed to the majority of the arterial thrombotic event reports for asthma drugs. The ranking distribution of the arterial thrombotic event categories was semihomogenous across the majority of asthma drug classes. Single-device combination ICS-LABA contributed to the majority of reports for angina, stroke, and cardiovascular death. Inhaled SABA contributed to the majority of reports for myocardial infarction, acute coronary syndrome, ischemic heart disease, and thrombosis. Among the exposure groups, omalizumab ranked third in cardiovascular death category; fourth in angina, myocardial infarction, acute coronary syndrome, and stroke categories; and fifth in ischemic heart disease and thrombosis categories.

\section{Serious arterial thrombotic events}

A serious adverse event is any unwanted medical incident associated with exposure to a medicinal product that, at any dose: results in patient death; is life-threatening; requires inpatient hospitalization or prolongs existing hospitalization; results in persistent or significant disability or incapacity; or is a congenital anomaly or birth defect. ${ }^{12}$ Overall, there were 1055 serious outcomes for all asthma drugs in AERS. Table 5 lists the distribution of serious arterial thrombotic event outcomes for asthma drugs. Arterial thrombotic events that required hospitalization after exposure to omalizumab accounted for $42 \%$ of all the serious arterial thrombotic event outcomes. About $16 \%$ of these serious events were attributed to mortality. Except for single-device combination ICS-LABA and xanthines, hospitalization accounted for the vast majority of serious arterial thrombotic event outcomes for asthma drugs. Single-device 


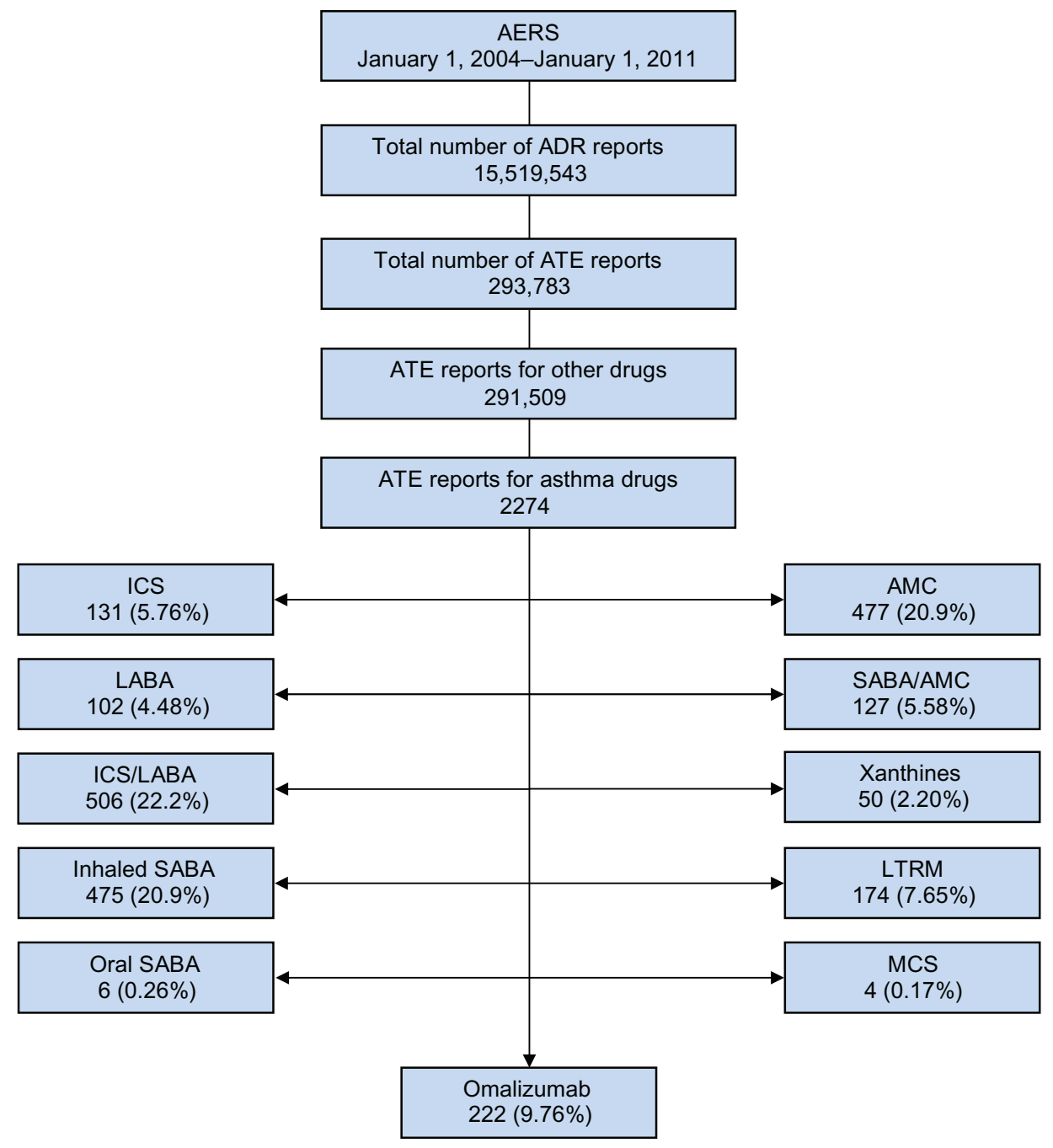

Figure I Study profile.

Abbreviations: AERS, Adverse Event Reporting System; ${ }^{8}$ ADR, adverse drug reactions; ATE, arteriothrombotic events; ICS, inhaled corticosteroids; LABA, long-acting beta-agonists; SABA, short-acting beta-agonists; AMC, antimuscarinics; LTRM, leukotriene modifiers; MCS, mast cell stabilizers.

combination ICS-LABA contributed to the majority of the mortality, life-threatening, and other unspecified serious arterial thrombotic event outcomes. Inhaled AMC contributed to the majority of serious disability and hospitalization outcomes. On the other hand, the preponderance of arterial thrombotic event outcomes that required intervention was for inhaled SABA. Compared with other asthma drugs, omalizumab ranked third in arterial thrombotic event outcomes that caused death, resulted in disability, and required hospitalization; seventh in lifethreatening arterial thrombotic events outcomes; and second in other unspecified serious arterial thrombotic events outcomes.

\section{Characteristics of arterial thrombotic event reports for omalizumab}

Table 6 shows the characteristics of arterial thrombotic event reports for omalizumab. The majority of omalizumab users who experienced arterial thrombotic events were females aged $\geq 45$ years. About $94 \%$ of the arterial thrombotic event reports were expedited reports, ie, reports for serious and unexpected events submitted by the manufacturer to the AERS within 15 days of occurrence. ${ }^{13}$ The product manufacturer is required to submit periodic reports for all adverse events quarterly for the first three years after product approval, and annually thereafter over multiple years. ${ }^{13}$ Periodic arterial thrombotic event reports for omalizumab accounted for $3 \%$ of the submitted arterial thrombotic event reports. Approximately $4 \%$ of the arterial thrombotic event reports were submitted directly by consumers or health care professionals. Fifty-five percent of the reports were initially submitted to the AERS, and $45 \%$ were classified as follow-up reports. The majority of arterial thrombotic event reports were received from within the US. Of the 
Table 4 Distribution of arterial thrombotic events across exposure groups

\begin{tabular}{|c|c|c|c|c|c|c|c|c|}
\hline \multirow[t]{2}{*}{ Drug } & \multicolumn{8}{|c|}{ Arterial thrombotic events, $\mathbf{n}(\%)^{*}$} \\
\hline & Angina & MI & ACS & Stroke & IHD & THR & CV death & Total \\
\hline Omalizumab & $12(5.4)$ & $65(29.3)$ & $4(1.8)$ & $60(27.0)$ & $40(18.0)$ & II (5.0) & $30(13.5)$ & 222 \\
\hline ICS & $10(7.6)$ & $43(32.8)$ & $\mathrm{I}(0.7)$ & $35(26.7)$ & $24(18.3)$ & $10(7.6)$ & $8(6.10)$ & $|3|$ \\
\hline LABA & $9(8.8)$ & $35(34.3)$ & $4(3.9)$ & $17(16.7)$ & $18(17.6)$ & $6(5.9)$ & $13(12.7)$ & 102 \\
\hline ICS-LABA & $59(11.6)$ & $|3|(25.9)$ & $8(1.6)$ & $127(25.1)$ & $66(13.0)$ & $27(5.3)$ & $88(17.4)$ & 506 \\
\hline SABA & $50(10.5)$ & $166(35.0)$ & $10(2.1)$ & $97(20.4)$ & $114(24.0)$ & $31(6.5)$ & $7(1.4)$ & 475 \\
\hline Oral SABA & $2(33.3)$ & $2(33.3)$ & 0 & 0 & 0 & $2(33.3)$ & 0 & 6 \\
\hline AMC & $48(10.0)$ & $160(33.5)$ & $3(0.6)$ & $104(21.8)$ & $65(13.6)$ & $20(4.2)$ & $77(16.1)$ & 477 \\
\hline SABA-AMC & $4(3.1)$ & $43(33.9)$ & 0 & 39 (30.7) & $27(21.3)$ & $7(5.5)$ & $7(5.5)$ & 127 \\
\hline Xanthines & $3(6.0)$ & $15(30.0)$ & $2(4.0)$ & $13(26.0)$ & $9(18.0)$ & $5(10.0)$ & $3(6.0)$ & 50 \\
\hline Leukotriene modifiers & $2(1.1)$ & $41(23.5)$ & $6(3.4)$ & $44(25.3)$ & $60(34.4)$ & $15(8.6)$ & $6(3.4)$ & 174 \\
\hline Mast cell stabilizers & I $(25.0)$ & $2(50.0)$ & 0 & 0 & 0 & I (25.0) & 0 & 4 \\
\hline Asthma drugs & $200(8.8)$ & $703(31.0)$ & $38(1.6)$ & $536(23.5)$ & $423(18.6)$ & $135(6.0)$ & $239(10.5)$ & 2274 \\
\hline Other drugs & $15,790(5.4)$ & $98,929(10.7)$ & $2554(0.8)$ & $76,594(26.3)$ & $51,859(17.8)$ & $26,027(9.0)$ & $19,756(6.6)$ & 291,509 \\
\hline Total & 15,990 & 99,632 & 2592 & 77,130 & 52,282 & 26,162 & 19,995 & 293,783 \\
\hline
\end{tabular}

Note: *Percentage of total arterial thrombotic events for the reported drug.

Abbreviations: MI, myocardial infarction; ACS, acute coronary syndrome; ICS, inhaled corticosteroids; IHD, ischemic heart disease; THR, thrombosis; CV, cardiovascular; AMC, antimuscarinics; ICS, inhaled corticosteroids; LABA, long-acting beta-agonists; SABA, short-acting beta-agonists.

eight cases reporting dechallenge information, four reported that arterial thrombotic events did abate when omalizumab was stopped. Only one case reported rechallenge information, which indicated that arterial thrombotic events did not relapse after reinstating omalizumab. The other reports stated that experimental information did not apply or was unknown if arterial thrombotic events terminated when dechallenge ensued, or recurred when rechallenge was carried out. About $97 \%$ of the reports classified omalizumab as the primary suspect in arterial thrombotic event occurrence. The majority of the arterial thrombotic event reports for omalizumab were submitted by physicians (52\%), followed by omalizumab users (28\%). Pharmacists accounted for $2 \%$ of these reports, whereas $19 \%$ of the reports were submitted by other health care professionals such as nurses. About $80 \%$ of the arterial thrombotic event reports were electronically submitted though the MedWatch online reporting portal; ${ }^{14}$ the remaining reports were sent via postal mail. About $94 \%$ of the cases reported that the patients experienced arterial thrombotic events more than two weeks after omalizumab use; only $6 \%$ of the reports stated that arterial thrombotic events occurred within two weeks of exposure. Although the majority of arterial thrombotic event reports were classified as expedited, $95 \%$ of the cases were reported more than two weeks after onset of the arterial thrombotic event. There was a trend of reporting arterial thrombotic event episodes for

Table 5 Distribution of serious arterial thrombotic events across exposure groups

\begin{tabular}{|c|c|c|c|c|c|c|c|}
\hline \multirow[t]{2}{*}{ Drug } & \multicolumn{7}{|c|}{ Serious arterial thrombotic events, $\mathbf{n}(\%)^{*}$} \\
\hline & Death & Disability & Hospital stay & Life-threatening & Required intervention & Other ${ }^{\S}$ & Total \\
\hline Omalizumab & $30(16.3)$ & $10(5.4)$ & $78(42.4)$ & I $(0.54)$ & 0 & $65(35.3)$ & 184 \\
\hline ICS & $8(29)$ & 0 & $10(35.7)$ & $2(7.1)$ & I (3.6) & $7(25)$ & 28 \\
\hline LABA & $13(27)$ & $3(6.3)$ & $21(44)$ & $2(4.2)$ & 0 & $9(19)$ & 48 \\
\hline ICS-LABA & $88(33.5)$ & $2(0.76)$ & $79(30)$ & II (4.2) & $\mathrm{I}(0.4)$ & $82(3 \mid .2)$ & 263 \\
\hline SABA & $7(4.5)$ & $10(6.5)$ & $72(46.5)$ & $4(2.6)$ & $4(2.6)$ & $58(37.4)$ & 155 \\
\hline Oral SABA & 0 & 0 & $3(60)$ & I (20) & 0 & I (20) & 5 \\
\hline AMC & $77(29)$ & $22(8.3)$ & $119(45)$ & $8(3)$ & I $(0.4)$ & $38(14.3)$ & 265 \\
\hline SABA-AMC & $7(16.3)$ & I (2.3) & $18(42)$ & $3(7)$ & 0 & $14(32.6)$ & 43 \\
\hline Xanthines & $3(43)$ & 0 & $2(12)$ & $I(6)$ & 0 & $11(65)$ & 17 \\
\hline Leukotriene modifiers & $6(13)$ & I (2.2) & $19(4 \mid .3)$ & $6(13)$ & 0 & $14(30.4)$ & 46 \\
\hline Mast cell stabilizers & 0 & 0 & 0 & 0 & 0 & $I(100)$ & 1 \\
\hline Asthma drugs & 239 & 49 & 421 & 39 & 7 & 300 & 1055 \\
\hline Other drugs & 19,756 & 7984 & 50,275 & 4314 & 816 & 46,379 & 129,524 \\
\hline Total & 19,995 & 8033 & 50,696 & 4353 & 823 & 46,679 & 130,579 \\
\hline
\end{tabular}

Notes: *Percentage of total serious events for the reported drug. Number of reports is mutually not exclusive, where one report could have more than one serious outcome classification; sunspecified serious outcomes are classified as "other". There were only 73 reports for congenital anomalies, which were for nonasthma drugs. Abbreviations: AMC, antimuscarinics; ICS, inhaled corticosteroids; LABA, long-acting beta-agonists; SABA, short-acting beta-agonists. 
Table 6 Characteristics of arterial thrombotic event reports for omalizumab

\begin{tabular}{|c|c|}
\hline Characteristic & n (\%) \\
\hline \multicolumn{2}{|l|}{ Sex } \\
\hline Female & $104(52)$ \\
\hline Male & $96(48)$ \\
\hline \multicolumn{2}{|l|}{ Age (years) } \\
\hline $13-44$ & $26(18.8)$ \\
\hline $45-64$ & $57(4 I .3)$ \\
\hline $65+$ & $55(39.9)$ \\
\hline \multicolumn{2}{|l|}{ Report type } \\
\hline Direct & $8(3.6)$ \\
\hline Expedited & $208(93.7)$ \\
\hline Periodic & $6(2.7)$ \\
\hline \multicolumn{2}{|l|}{ Report status } \\
\hline Initial & $122(55)$ \\
\hline Follow-up & $100(45)$ \\
\hline \multicolumn{2}{|l|}{ Report source } \\
\hline Domestic & $119(53.6)$ \\
\hline Foreign & $103(46.4)$ \\
\hline \multicolumn{2}{|l|}{ Experimental dechallenge } \\
\hline Positive result & $4(50)$ \\
\hline Negative result & $4(50)$ \\
\hline \multicolumn{2}{|l|}{ Experimental rechallenge } \\
\hline Negative result & $I(100)$ \\
\hline \multicolumn{2}{|l|}{ Drug role in ATE occurrence } \\
\hline Primary suspect & $206(97.2)$ \\
\hline Secondary suspect & $6(2.8)$ \\
\hline \multicolumn{2}{|l|}{ Reporter occupation } \\
\hline Consumer & $57(27.8)$ \\
\hline Physician & $106(51.7)$ \\
\hline Pharmacist & $4(2.0)$ \\
\hline Other health care professional & $38(18.5)$ \\
\hline \multicolumn{2}{|l|}{ Report submission mode } \\
\hline Electronic & I 77 (79.7) \\
\hline Mail & $45(20.3)$ \\
\hline \multicolumn{2}{|l|}{ Time to ATE onset (days) } \\
\hline $0-14$ & $7(6.1)$ \\
\hline$>14$ & $107(93.9)$ \\
\hline \multicolumn{2}{|c|}{ Time to report ATE since onset (days) } \\
\hline $0-14$ & $7(5.0)$ \\
\hline$>14$ & $134(95)$ \\
\hline \multicolumn{2}{|l|}{ Reporting year } \\
\hline 2004 & $4(1.8)$ \\
\hline 2005 & $17(7.7)$ \\
\hline 2006 & $12(5.4)$ \\
\hline 2007 & $22(10)$ \\
\hline 2008 & $19(8.5)$ \\
\hline 2009 & 74 (33.3) \\
\hline 2010 & 74 (33.3) \\
\hline
\end{tabular}

Abbreviation: ATE, arteriothrombotic events.

omalizumab, which was steadily increasing, especially for 2004-2005 and 2008-2010.

\section{Reporting odds ratio data mining algorithm results}

Figure 2 depicts the reporting odds ratio and corresponding 95\% CI for omalizumab and other asthma drugs. Compared with other asthma drugs, omalizumab ranked second after ICS in showing a statistically significant safety signal in relation to arterial thrombotic events. Exposure to omalizumab was associated with higher than expected reporting of arterial thrombotic events (reporting odds ratio 2.75, 95\% CI 2.39-3.16). A similar analysis was conducted using all the drugs in the dataset as a comparison group, including other asthma drugs, which yielded more conservative estimates for omalizumab, albeit not statistically significant (reporting odds ratio 1.09, 95\% CI 0.95-1.24). Signal dilution was ensued when all the drugs in the database were used as a comparator. Figure 3 illustrates the data mining estimates for selected arterial thrombotic events for omalizumab, which are calculated in comparison with other asthma drugs in the AERS. Cardiovascular death and stroke ranked first on the arterial thrombotic events list in detection of statistically significant safety signals (reporting odds ratio 3.14, 95\% CI 2.14-4.56; reporting odds ratio 3.14, 95\% CI 2.41-4.05, respectively). Although the reporting odds ratio values for ischemic heart disease, myocardial infarction, and thrombosis risk exceeded 2.0, the lower limit of the 95\% CI failed to reach that threshold, making the estimates less significant as safety signals (reporting odds ratio 2.63, 95\% CI 1.92-3.63; reporting odds ratio 2.55, 95\% CI 1.98-3.29; reporting odds ratio $2.19,95 \%$ CI $1.18-4.01$, respectively). The data mining algorithm did not show a statistically significant result for angina (reporting odds ratio 1.60, 95\% CI 0.89-2.86). Albeit not statistically significant, the analysis showed that omalizumab was associated with less than expected reporting of acute coronary syndrome compared with other asthma drugs (reporting odds ratio $0.53,95 \%$ CI $0.20-1.42$ ).

\section{Discussion}

Adverse event reports submitted to the FDA AERS database from January 1, 2004 to January 1, 2011 were used to conduct a retrospective pharmacovigilance analysis aimed at evaluating the association of omalizumab with arterial thrombotic events, which yielded a statistically significant safety signal for the association of omalizumab with arterial thrombotic events relative to other asthma drugs. Myocardial infarction $(n=65)$ and stroke $(n=60)$ accounted for the majority of the omalizumab arterial thrombotic event reports. However, cardiovascular death and stroke events were associated with the strongest safety signals for omalizumab (Figure 3 ). These findings are consistent with the results from clinical trials and EXCELS cohort study. ${ }^{4,6}$ However, risk estimates from these studies were not statistically significant despite the large sample sizes; this could be attributed to the fact that these studies were not powered to detect arterial thrombotic events 


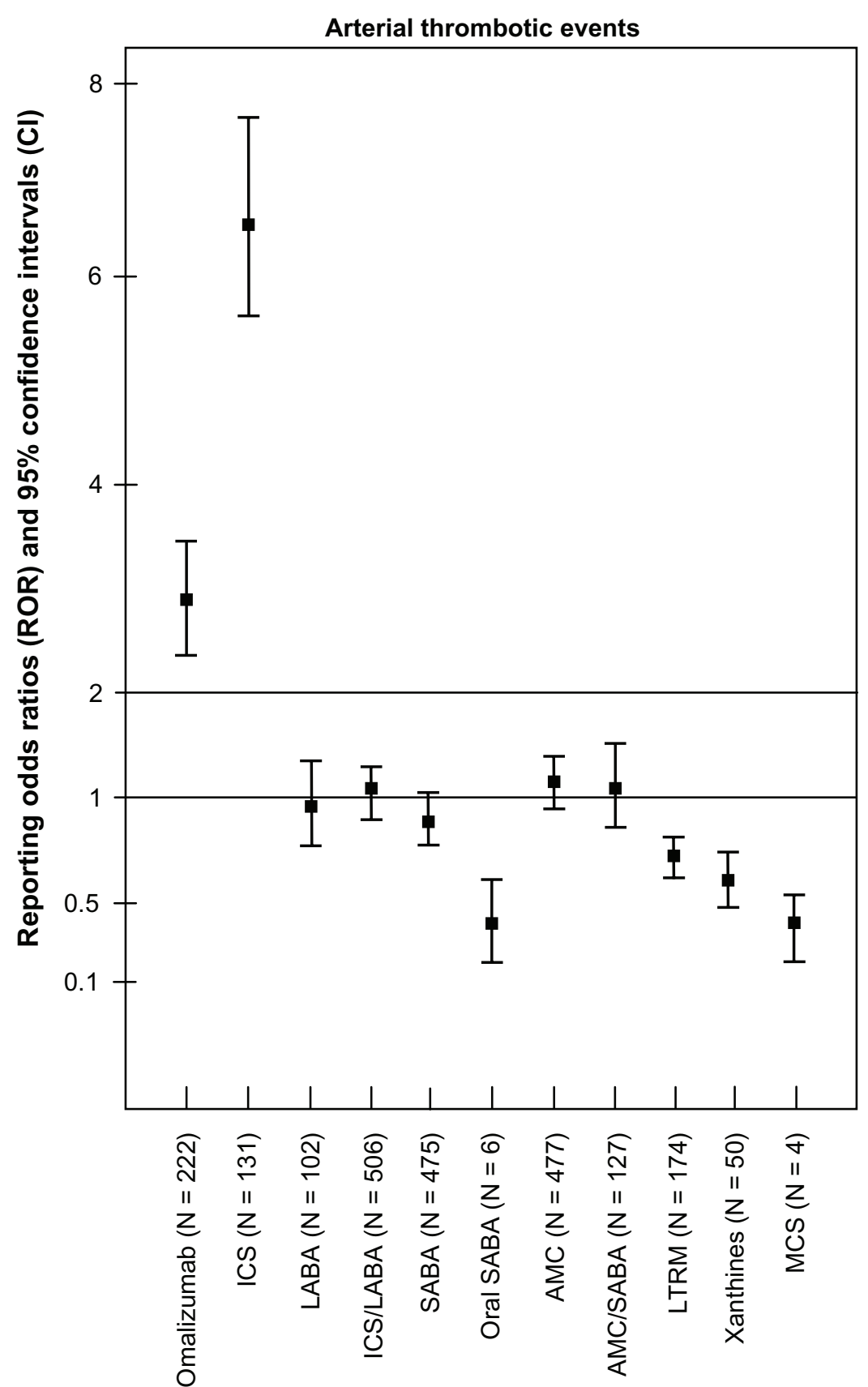

Figure 2 Reporting odds ratios and $95 \%$ confidence intervals for arterial thrombotic events by asthma medication classes.

Abbreviations: ICS, inhaled corticosteroids; LABA, long-acting beta-agonists; SABA, short-acting beta-agonists; AMC, antimuscarinics; LTRM, leukotriene modifiers; MCS, mast cell stabilizers.

as an outcome of interest. ${ }^{4}$ According to our knowledge, the current pharmacovigilance analysis is the first to detect such a safety signal in a spontaneous adverse event reporting database. However, such analysis has several limitations that are inherent in the nature of the data, which require consideration prior to drawing conclusions about such findings. Uncertain quality of reported cases, missing information, variable reporting, and under-reporting of adverse events are common features of spontaneous reporting systems. Under-reporting in spontaneous reporting systems is attributed to reasons that range from mere ignorance of adverse event reporting requirements, the misconception that only safe products are approved for marketing, to fear of involvement in litigation. ${ }^{15}$ Furthermore, reporting rates for specific adverse events or products vary over time. This is contingent upon the length of time the product is on the market, where newer products have more frequent reports, publicity about the product or the adverse event, marketing and promotion for the product, and indication or contraindication 


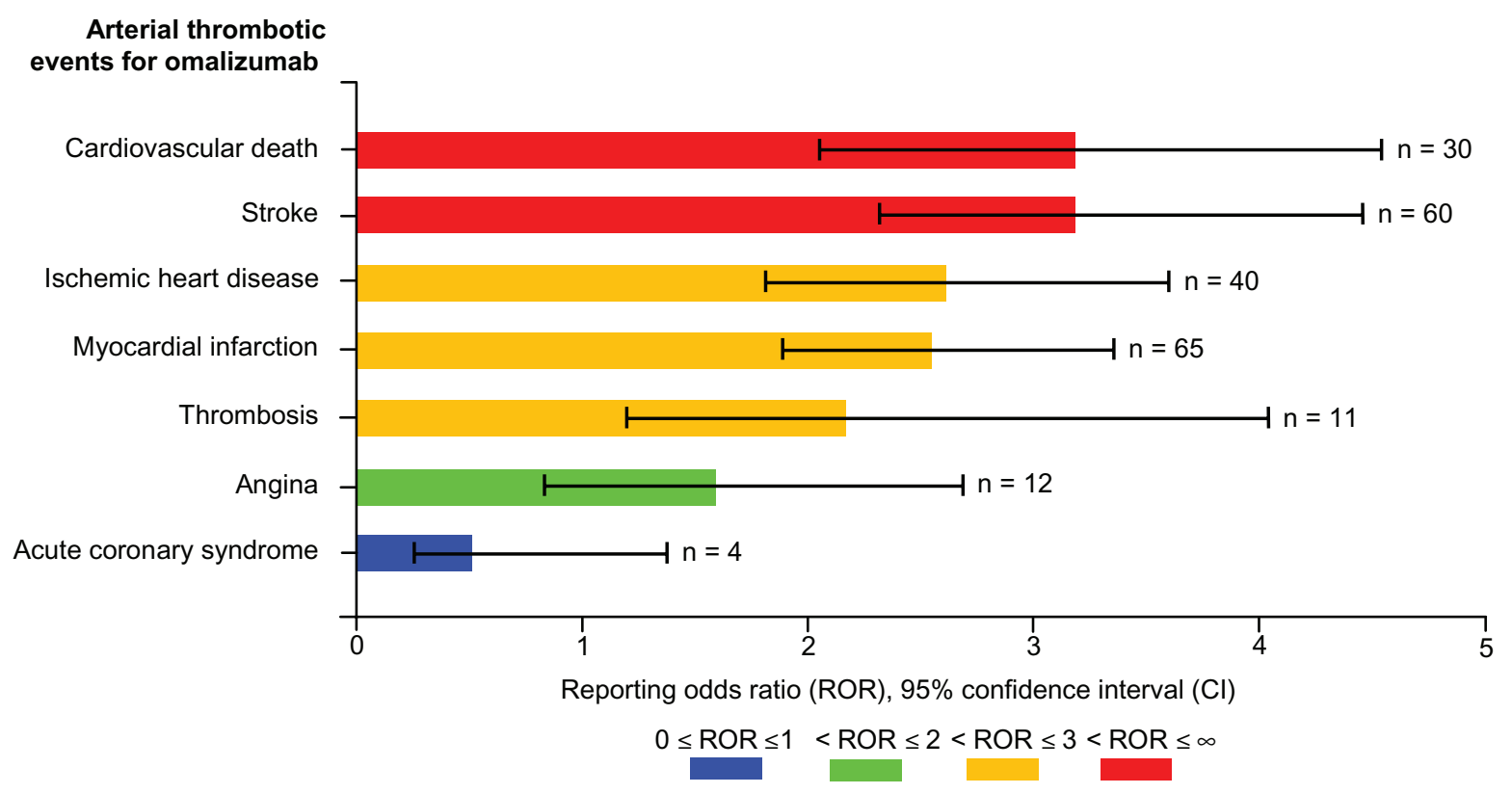

Figure 3 Visual presentation of reporting odds ratios and $95 \%$ confidence intervals for selected arterial thrombotic events for omalizumab.

information for the product. Nevertheless, the potential for confounding and misinterpretation of signals as incidence estimates are the most common drawbacks of pharmacovigilance analyses using spontaneous reporting systems. Limited information about event temporality, concurrent morbidity, and concomitant medications introduces confounding. For example, risk factors that contribute to arterial thrombotic events occurrence are not recorded in AERS, including obesity, history of diabetes, hypertension, and hypercholesterolemia. ${ }^{16}$ Association estimates from the data mining algorithm should not be interpreted as incidence measures, rather more than expected reporting of an adverse event for a specific product. Therefore, the potential for Type I error when performing multiple statistical tests is high; the statistical significance of these findings is overestimated, and further studies are warranted to follow such clinically significant associations. ${ }^{11}$

\section{Conclusion}

Exposure to omalizumab is associated with an increased risk of arterial thrombotic events in asthmatic patients, predominantly cardiovascular death and stroke. In light of causality restraints of the current analysis, robust epidemiological studies are recommended to test this hypothesis further in order to draw conclusions that contribute to clinical practice and public health. Meanwhile, until such conclusions are made and recommendations are prepared by regulatory authorities, the findings suggest that prescribers should cautiously prescribe omalizumab to asthmatic patients who have risk factors that contribute to arteriothrombotic events.

\section{Acknowledgment}

The authors thank Teba Mohammad for reviewing the final draft of the manuscript. Publication of this article was funded in part by the University of Florida Open-Access Publishing Fund.

\section{Disclosure}

The authors report no conflicts of interest in this work.

\section{References}

1. Buhl R. Anti-IgE antibodies for the treatment of asthma. Curr Opin Pulm Med. 2005;11(1):27-34.

2. Food and Drug Administration. FDA approved drug products (Drugs @ FDA), updated August 2011. Available from: http://www.accessdata. fda.gov/scripts/cder/drugsatfda/index.cfm. Accessed August 3, 2011.

3. American Society for Health-System Pharmacists. AHFS Drug Information Essentials: 2004-2005. Omalizumab. Bethesda, MD: American Society for Health-System Pharmacists; 2004.

4. Medicines and Healthcare Regulatory Agency. Omalizumab: Potential risk of arterial thrombotic events. Available from: http://www.mhra.gov. uk/home/groups/dsu/documents/publication/con108718.pdf. Accessed August 3, 2011.

5. World Health Organization. Omalizumab: Potential risk of arterial thrombotic events. 2011. Available from: http://www.who.int/ medicines/publications/Newsletter_2-2011.pdf. Accessed August 3, 2011.

6. Long AA, Fish JE, Rahmaoui A, et al. Baseline characteristics of patients enrolled in EXCELS: a cohort study. Ann Allergy Asthma Immunol. 2009; 103(3):212-219.

7. Food and Drug Administration. Postmarket drug safety information for patients and providers. Early communication about an ongoing safety review of omalizumab (marketed as Xolair). July 16, 2009. Available from: http://www.fda.gov/Drugs/DrugSafety/PostmarketDrugSafety InformationforPatientsandProviders/DrugSafetyInformationforHeath careProfessionals/ucm172218.htm. Accessed August 3, 2011. 
8. Food and Drug Administration. Adverse Event Reporting System (AERS): Latest quarterly data files. April 18, 2011. Available from: http://www.fda.gov/Drugs/GuidanceComplianceRegulatoryInforma tion/Surveillance/AdverseDrugEffects/ucm082193.htm. Accessed May 12, 2011.

9. Ali AK. Pharmacovigilance analysis of adverse event reports for aliskiren hemifumarate, a first-in-class direct renin inhibitor. Ther Clin Risk Manag. 2011;7:337-344.

10. Clinical Pharmacology [online database]. Tampa, FL: Gold Standard Inc, 2011. Available from: http://www.clinicalpharmacology.com. Updated August 2011. Accessed March 29, 2012.

11. Bate A, Edwards IR. Data mining techniques in pharmacovigilance. In: Hartzema AG, Tilson HH, Chan KA, editors. Pharmacoepidemiology and Therapeutic Risk Management. 1st ed. Cincinnati, OH: Harvey Whitney Books Co; 2008.

12. Food and Drug Administration. CFR-Code of Federal Regulations Title 21. Updated April 1, 2011. Available from: http://www.accessdata. fda.gov/scripts/cdrh/cfdocs/cfcfr/CFRSearch.cfm?fr=312.32. Accessed November 11, 2011.
13. Food and Drug Administration. Staff manual guide: Chapter 53; Postmarketing surveillance and epidemiology: human drugs. Adverse drug effects. Updated May 12, 2010. Available from: http://www.fda. gov/Drugs/GuidanceComplianceRegulatoryInformation/Surveillance/ ucm129115.htm. Accessed November 11, 2011.

14. Food and Drug Administration. MedWatch: The FDA Safety Information and Adverse Event Reporting Program. MedWatch Online Voluntary Reporting Form (3500). Updated October 5, 2011. Available from: https://www.accessdata.fda.gov/scripts/medwatch/medwatch-online. htm. Accessed November 13, 2011.

15. Inman WHW. Detection and investigation of drug safety problems. In: Gent M, Shigamatsu I, editors. Epidemiological Issues in Reported Drug-Induced Illnesses. Ontario, Canada: McMaster University Library Press; 1978.

16. Prandoni P. Venous and arterial thrombosis: two aspects of the same disease? Clin Epidemiol. 2009;1:1-6.

\section{Publish your work in this journal}

The Journal of Asthma and Allergy is an international, peer-reviewed open-access journal publishing original research, reports, editorials and commentaries on the following topics: Asthma; Pulmonary physiology; Asthma related clinical health; Clinical immunology and the immunological basis of disease; Pharmacological interventions and

\section{Dovepress}

new therapies. Issues of patient safety and quality of care will also be considered. The manuscript management system is completely online and includes a very quick and fair peer-review system, which is all easy to use. Visit http://www.dovepress.com/testimonials.php to read real quotes from published authors.

Submit your manuscript here: http://www.dovepress.com/journal-of-asthma-and-allergy-journal 